\title{
Enhancing Cosmesis While Achieving High Cure- Rates For Early-Stage Non-Melanoma Skin Cancer In The Outpatient Dermatology Clinic Using Novel Non- Invasive Modality
}

\author{
Lio Yu ${ }^{1}$, Mairead Moloney ${ }^{2 *}$, Raymond Beers ${ }^{3}$ and Donna Serure ${ }^{4}$ \\ ${ }^{1}$ Director of Radiation Oncology, Laserderm Dermatology, USA \\ ${ }^{2}$ New York Institute of Technology College of Osteopathic Medicine, Old Westbury, USA \\ ${ }^{3}$ Astarita Associates, USA \\ ${ }^{4}$ Chief of Dermatology, Laserderm Dermatology, USA
}

*Corresponding author: Mairead Moloney, BS, OMS-II, New York Institute of Technology College of Osteopathic Medicine, South Setauket, NY 11720, USA.

To Cite This Article: Lio Yu, Mairead Moloney, Raymond Beers, Donna Serure. Enhancing Cosmesis While Achieving High Cure-Rates For Early-Stage Non-Melanoma Skin Cancer In The Outpatient Dermatology Clinic Using Novel Non-Invasive Modality. Am J Biomed Sci \& Res. 2021 - 12(6). AJBSR.MS.ID.001803. DOI: 10.34297/AJBSR.2021.12.001803.

Received: 䟧 February 13, 2021; Published: 䟧 May 07, 2021

\section{Abstract}

Introduction: Non-Melanoma Skin Cancer (NMSC) is generally treated in dermatology offices using surgical techniques.

Objectives: We evaluate the feasibility, tolerance, effectiveness and cosmesis using an office-based non-surgical modality for NMSC.

Methods: 93 patients with 133 pathologically confirmed early stage invasive and in-situ NMSC lesions treated with Image-Guided Superficial Radiation Therapy (IGSRT) were retrospectively analyzed. All lesions received a median of twenty fractions of 50 or 70 kilovoltage(kV) IGSRT. Energy selection and subsequent $\mathrm{kV}$ changes were determined by ultrasound imaging. RTOG toxicity scoring was used. Treatment interruption was defined as greater than 2 weeks.

Results: Median age was 69. At an average follow-up of 16.23 months, 92 of 93 patients were alive. One patient expired from unrelated causes while no evidence of disease (NED). 132 of 133 lesions achieved local control (LC) with one lesion recurring at 12.9 months. Absolute LC was $99.2 \%$, overall Kaplan-Meier LC (KM LC) was $98.95 \%$ at 30.8 months, and Disease Free Survival (DFS) was 100\%. Acute toxicities were mild with RTOG grades 0,1 or 2 in all lesions with no grade 3 or 4 toxicity. Cosmesis was felt to be excellent or very good (VG) by the clinicians with no fair/poor cosmesis. There were no severe toxicities or complications requiring treatment interruptions.

Conclusion: Office based IGSRT is feasible, safe, easily tolerable, and highly effective. Patients receiving IGSRT achieve excellent/VG cosmesis and welcome this nonsurgical option. IGSRT is an attractive non-invasive therapeutic option for NMSC and provides another valuable tool for dermatologists.

Keywords: Image-guided superficial radiation therapy; Non-melanoma skin cancer; Basal cell carcinoma; Squamous cell carcinoma; Squamous cell carcinoma in situ

Abbreviations: AAD: American Academy of Dermatology; ADLs: Activities of Daily Living; AJCC: American Joint Committee on Cancer; ASTRO: American Society for Radiation Oncology; BCC: Basal Cell Carcinoma; cGy: Centigray: cm: Centimeter; cSCC: Cutaneous Squamous Cell Carcinoma; DFS: Disease Free Survival; ED\&C: Electrodessication and Curettage; FTA: Full Thickness Atypia; IGSRT: Image-Guided Superficial Radiation Therapy; KM: Kaplan-Meier; kV: Kilovoltage; LC: Local Control; LLE: Limited Life Expectancy; mm: Millimeter; MMS: Mohs Micrographic Surgery; NCCN: National Comprehensive Cancer Network; NED: No Evidence of Disease; NMSC: Non-Melanoma Skin Cancer; RCT: Randomized Controlled Trial; RT: Radiation Therapy; RTOG: Radiation Treatment Oncology Group; SCC: Squamous Cell Carcinoma; SCCIS: Squamous Cell Carcinoma in situ; SD: Standard Deviation; SRT: Superficial Radiation Therapy; TDF: Time Dose Fractionation; VG: Very Good 


\section{Introduction}

The incidence of skin cancer is on the rise for both melanoma and non-melanoma skin cancer (NMSC) with NMSC being 18 to 20 times higher than melanoma [1]. NMSC is the most prevalent cancer in the United States with the current estimate indicating 3.3 million patients with 5.43 million lesions in 2012 [2,3]. NMSC comprise of Basal Cell Carcinoma (BCC) and Squamous Cell Carcinoma (SCC), which are keratinocyte carcinomas, whereas melanoma is a melanocyte carcinoma [4]. BCC and SCC account for 99\% of NMSC, with BCC making up $80 \%$ of skin cancers $[1,4,5]$. In the United States, there are between 5.03 to 5.23 million BCC lesions diagnosed each year and there are between 200,000 to 400,000 cases of SCC each year [6,7]. Major risk factors for the development of NMSC include duration and intensity of sun exposure, increased age and fair skin, which is why Caucasians over 65 are at the greatest risk for developing skin cancer [5,8-10]. The greatest incidence of NMSC occurs in sun exposed areas including the head and neck. Consequently it is imperative that we identify treatment modalities that result in high cure rates and superior cosmesis [5,7].

Despite the high occurrence of NMSC, they are considered nonfatal and curable due to their slow growth, low recurrence, and rare metastasis with limited impact on mortality and morbidity $[9,10]$. However, it is standard that NMSC are treated to prevent invasion and tumor growth [4,5]. Additionally, small superficial cutaneous squamous cell carcinoma, if left untreated, can locally invade and lead to metastasis and potential mortality. Treatment modalities include Mohs micrographic surgery (MMS), surgical excision, radiation therapy, electrodessication and curettage (ED\&C), cryotherapy, laser, photodynamic therapy, topical medications and systemic medications $[2,4,9,10]$. Although there are a variety of less invasive non-surgical options available for the treatment of NMSC, surgery remains the standard for NMSC treatment, specifically Mohs micrographic surgery [5]. This is due to the fact that current evidence suggests MMS offers the highest cure rates, with about a 1\% 5-year recurrence for BCC and less than 6\% 5-year recurrence for SCC [11,12]. A drawback of MMS for NMSC is that in anatomical locations where conservation of tissue is important, such as the ear, nose, eyelid and neck, MMS removes tissue layers posing cosmetic concerns $[4,5,7,8]$.

Another concern is that a majority of NMSC occur in older patients, who might not be able to endure the length of time in the surgical position MMS requires to excise the skin cancer in addition to the sequela that follow after surgery $[9,11]$. Linos et al. [9] reported that patients with a limited life expectancy (LLE) may not benefit from surgical removal of NMSC when compared to patients who received non-surgical treatment or no treatment. Individuals with LLE typically have difficulty with activities of daily living (ADLs) and would likely need assistance with wound care after surgery [5].
A non-surgical treatment option would minimize the complications of skin surgery, such as infection, excessive bleeding, and wound dehiscence. Furthermore, a non-surgical approach is attractive to patients who are not medically surgical candidates, such as those with cardiac risk factors, on blood thinners, or otherwise precluded from having surgery. Nonsurgical options have the potential to maintain better cosmesis and function with minimal pain.

\section{Objective}

We report early results of a non-surgical modality for the treatment of NMSC - Image-Guided Superficial Radiation Therapy (IGSRT). This newer image guided treatment combines the use of ultrasound for non-invasive imaging and an established treatment of superficial radiation therapy, which has been used for decades [12].

\section{Methods}

\section{Patient selection}

We retrospectively analyzed the medical charts of 93 patients (60 female, 33 male) from an outpatient dermatology practice in Smithtown, NY. The authors adhered to the principles established in the Federal Policy for the Protection of Human Subjects, referred to as the "Common Rule," as well as the pertinent sections of the Helsinki Declaration and its amendments. The data have been deidentified for use in this study.

Patients with pathologically confirmed early stage (Stage 0, I, II) NMSC lesions, specified by 8th edition of the American Joint Committee on Cancer (AJCC) Staging Manual [13] treated with IGSRT between April 2017 and August 2018 were analyzed. All BCC and non-head and neck cutaneous SCC ( $\mathrm{CSCC}$ ) that fell outside of AJCC staging (which currently only include head and neck cSCC) were included and staging was extrapolated to those lesions for consistency. SCC In-Situ (SCCIS) lesions were only selected for treatment if they had full thickness atypia (FTA) deemed appropriate for treatment by NCCN guidelines [14]. Lesions from patients with multiple NMSC lesions treated synchronously or metachronously with IGSRT were included in the study for a total of 133 lesions. The histopathology and stage for all lesions were confirmed prior to IGSRT treatment. All patients signed informed consent prior to treatment.

\section{Treatment methods}

Patient's lesions were generally treated with 50, 70, or mixed (50 and 70) kiloVoltage (kV) energy IGSRT given 3 or 4 times weekly (range 2-4) (Table 1). Lesions were treated with a median of 20 fractions of 256 cGy (range 242 to 283 cGy) for a total cumulative dose of 5128 cGy over 1.58 months. The protocol used recommended a typical dose fractionation in the range of 245-265 cGy for 20 fractions as a routine and can vary somewhat based on 
tumor characteristics, size of treatment cone applicator, histology and depth of the lesion ultrasound. The IGSRT machine is not designed to deliver doses based on cGy dose but instead based on time (minutes) the beam is turned on to 2 decimal points. Therefore, the treatment dose that comes closest to that recommended by the protocol doses is generally selected. Energy selection and subsequent $\mathrm{kV}$ changes were determined by ultrasound imaging of the lesion and clinical exam characteristics. Doses were prescribed to the skin surface. Radiation Treatment Oncology Group (RTOG) toxicity scoring [15] was used throughout the treatment every 1 to 2 weeks generally after every 5 fractions. The highest RTOG score was recorded for this retrospective analysis. Treatment interruption was defined as an unplanned break due to side effects of greater than 2 weeks. Patients were generally followed initially 2-4 weeks after treatment completion and scheduled for continued followed-up until there was no evidence of disease (NED) or if failure occurred. Thereafter or contemporaneously, patients returned to their dermatologists for follow-up and management.

Table 1: Treatment characteristics for IGSRT (Image-Guided Superficial Radiation Therapy).

\begin{tabular}{|c|c|c|}
\hline \multicolumn{3}{|c|}{ IGSRT Treatment Characteristics } \\
\hline \multirow{4}{*}{$\begin{array}{l}\text { Total dose received } \\
\text { (cGy) }\end{array}$} & Mean & $\begin{array}{c}5128.53(\mathrm{SD}+/- \\
140.93)\end{array}$ \\
\hline & Median & 5128 \\
\hline & Minimum & 4783 \\
\hline & Maximum & 6032.57 \\
\hline \multirow{4}{*}{ Number of fractions } & Mean & $19.94(\mathrm{SD}+/-1.02)$ \\
\hline & Median & 20 \\
\hline & Minimum & 13 \\
\hline & Maximum & 24 \\
\hline \multirow{4}{*}{$\begin{array}{l}\text { Dose per fraction } \\
\text { (cGy) }\end{array}$} & Mean & $257.06(\mathrm{SD}+/-14.96)$ \\
\hline & Median & 256 \\
\hline & Minimum & 242 \\
\hline & Maximum & 383 \\
\hline \multirow{4}{*}{$\begin{array}{l}\text { Energy (kV) [Number } \\
\text { of lesions] }\end{array}$} & $50 \mathrm{kV}$ & $110(82.7 \%)$ \\
\hline & $70 \mathrm{kV}$ & $9(6.8 \%)$ \\
\hline & $100 \mathrm{kV}$ & $0(0 \%)$ \\
\hline & Mixed - $50 \mathrm{kV} \& 70 \mathrm{kV}$ & $14(10.5 \%)$ \\
\hline \multirow{4}{*}{$\begin{array}{l}\text { Treatment duration } \\
\text { (months) }\end{array}$} & Mean & $1.56(\mathrm{SD}+/-0.26)$ \\
\hline & Median & 1.58 \\
\hline & Minimum & 0.99 \\
\hline & Maximum & 2.83 \\
\hline \multirow{4}{*}{$\begin{array}{l}\text { Treatment duration } \\
\text { (weeks) }\end{array}$} & Mean & $6.82(\mathrm{SD}+/-1.12)$ \\
\hline & Median & 6.86 \\
\hline & Minimum & 4.29 \\
\hline & Maximum & 12.29 \\
\hline
\end{tabular}

\begin{tabular}{|c|c|c|}
\hline \multirow{4}{*}{$\begin{array}{c}\text { Time Dose } \\
\text { Fractionation }\end{array}$} & Mean & $88.35(\mathrm{SD}+/-3.23)$ \\
\cline { 2 - 3 } & Median & 88 \\
\cline { 2 - 3 } & Minimum & 81 \\
\cline { 2 - 3 } & Maximum & 105 \\
\hline
\end{tabular}

Board-certified Radiation Therapists administered each fraction based on the IGSRT prescription determined by the Radiation Oncologist. A Medical Physicist ensured that all calculations and treatment parameters were in accordance with the prescription, as well as verifying the accurate completion of the treatment course. The Medical Physicist also maintained quality and safety checks on the SRT machine on a regular basis.

\section{Statistical analysis}

Because follow-up time differed from patient to patient, we also used Kaplan-Meier analysis to calculate local control rates. Statistical differences were calculated using the log-rank test. P value $<0.05$ was considered statistically significant. Kaplan-Meier analysis and descriptive statistical analysis was performed with $\mathrm{R}$ Studio. Any missing data was not included in the statistical analyses.

\section{Results}

This study analyzed 93 patients with 133 lesions receiving IGSRT with a median age of 69 at first treatment (Table 2). 60 patients were female $(64.5 \%)$ and 33 patients were male (35.5\%). Average follow-up after IGSRT treatment was 16.23 months (SD +/- 8.88). Lesion characteristics are shown in Table 3. Of the total of 133 lesions, 67 were BCC, 17 were SCC, 49 were SCCIS with full thickness atypia. Forty-nine lesions were stage 0 (zero), 77 lesions were stage I, and 7 lesions were stage II. Median lesion size was $1.0 \mathrm{~cm}$. Seventy-four lesions had diameters greater than or equal to $1 \mathrm{~cm}$. Six lesions were greater than $2 \mathrm{~cm}$ in dimension with 3 of these lesions (all SCCIS) greater than $3 \mathrm{~cm}$ in dimension.

Table 2: Patient demographics at the time of treatment.

\begin{tabular}{|c|c|c|}
\hline \multicolumn{2}{|c|}{ Patient Characteristics } \\
\hline \multirow{2}{*}{ Total Patients } & 93 \\
\hline \multirow{3}{*}{\begin{tabular}{c} 
Age at $\begin{array}{c}\text { st } \\
\text { (years) } \\
\text { treatment }\end{array}$ \\
\cline { 2 - 3 }
\end{tabular}} & Mean & $68.72(\mathrm{SD}+/-10.9)$ \\
\cline { 2 - 3 } & Median & 69 \\
\cline { 2 - 3 } Gender & Maximum & 36 \\
\cline { 2 - 3 } & Female & $60(64.5 \%)$ \\
\hline \multirow{3}{*}{$\begin{array}{c}\text { Follow-up interval } \\
\text { (months) }\end{array}$} & Male & $33(35.5 \%)$ \\
\cline { 2 - 3 } & Mean & $16.23(\mathrm{SD}+/-8.88)$ \\
\cline { 2 - 3 } & Median & 0 \\
\cline { 2 - 3 } & Minimum & 30.8 \\
\hline
\end{tabular}




\section{Response}

Local control: Initially, all lesions achieved local control (LC) within 4 months. One lesion recurred at 12.9 months post treatment. Absolute local control (LC) rate was 99.2\%. Overall Kaplan-Meier (KM) LC was $98.95 \%$ at the maximum follow-up time of 30.8 months (2.57 years) (Figure 1). There was no significant difference in KM LC between stages (Log-rank $\mathrm{p}=0.6729$ ) (Figure 2). KM LC for stage 0 (SCCIS) is $100 \%$ at 12,24 and 30.8 months. KM LC for stage I is $100 \%$ at 12 months and remained at $98.11 \%$ for 24 and 30.8 months. KM LC for stage II remained at $100 \%$ at 12,24 and 30.8 months.

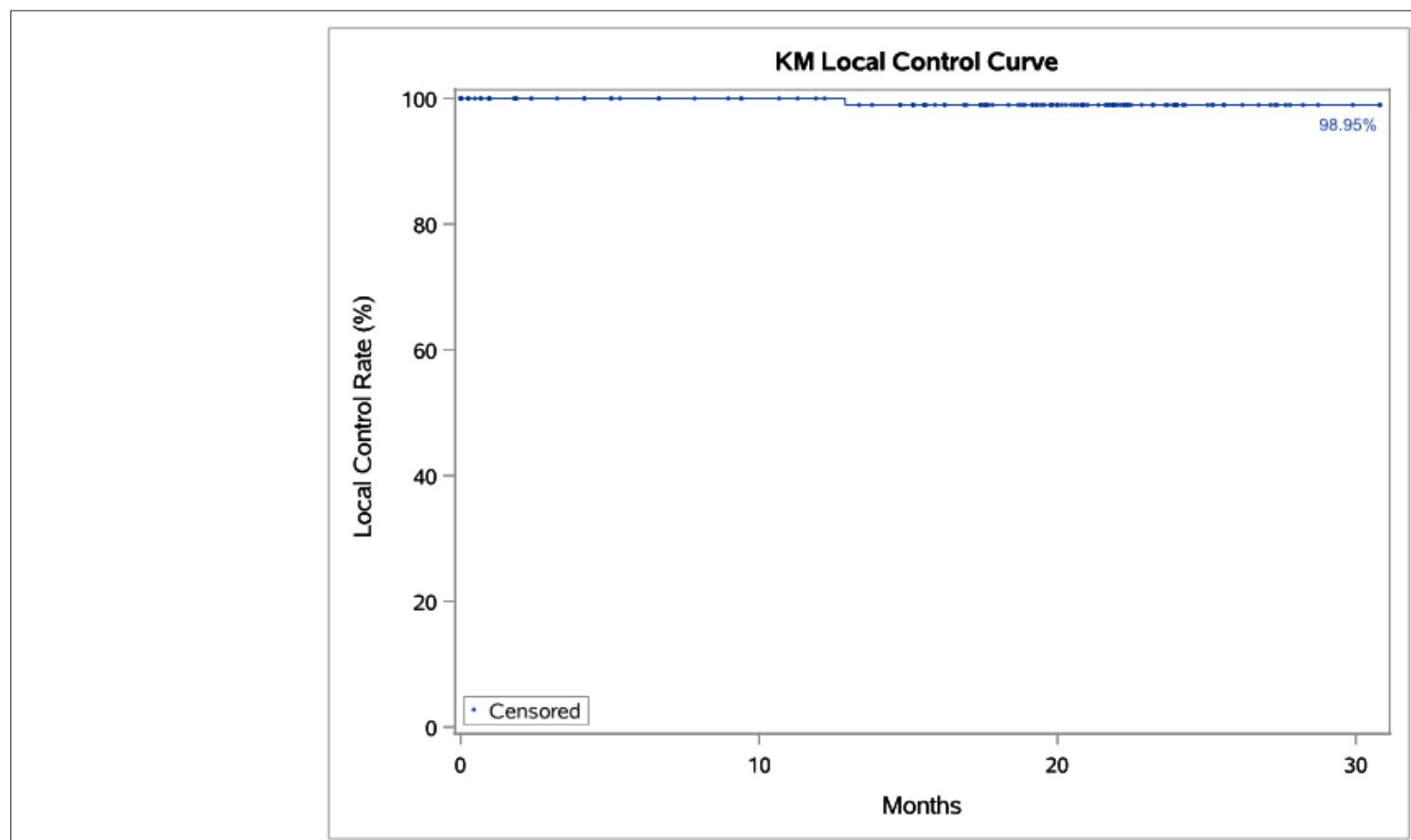

Figure 1: Kaplan-Meier (KM) Local control (LC) for 133 lesions (67 BCC, 17 SCC, 49 SCCIS) treated with IGSRT (Image-Guided Superficial Radiation Therapy). Dots represent censored events.

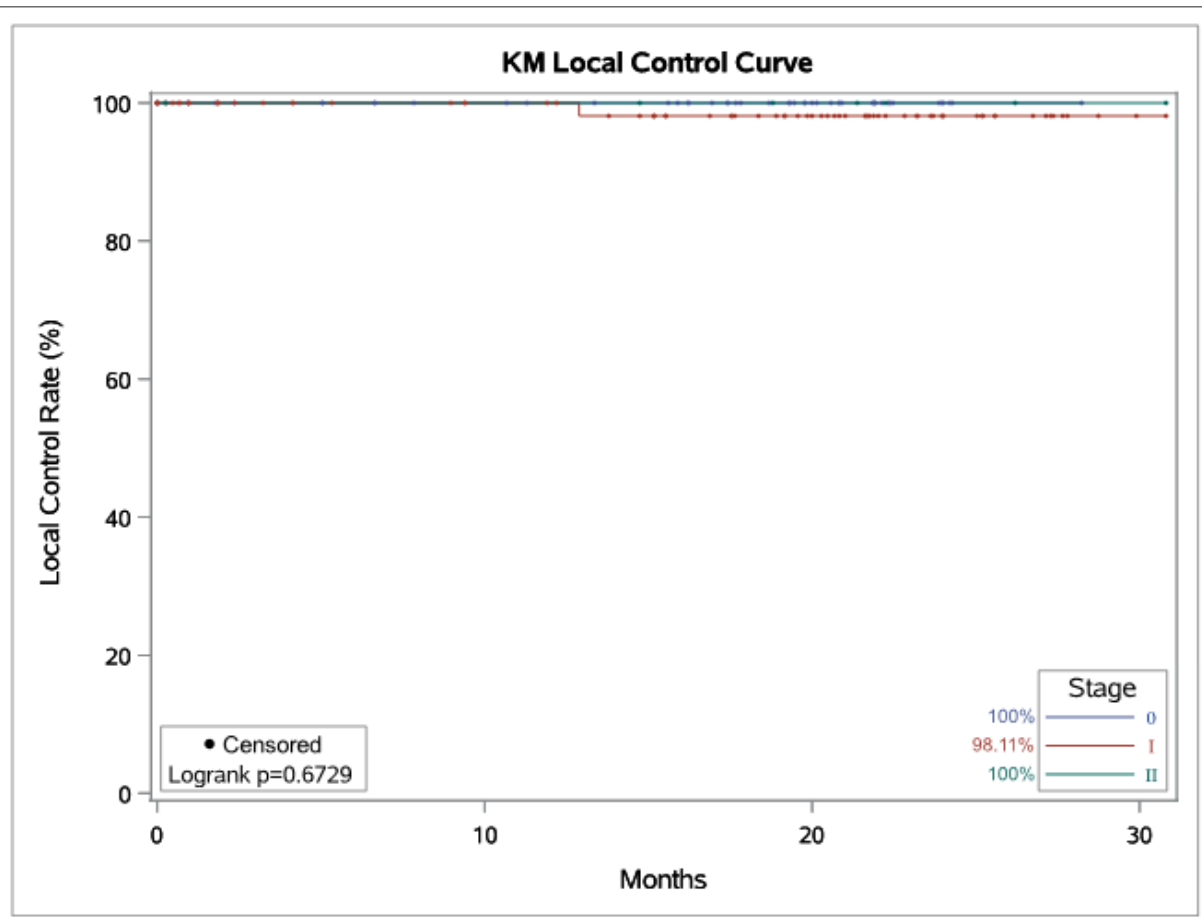

Figure 2: Kaplan-Meier (KM) Local control (LC) of 133 lesions (Stage $0=49, I=77, I=7$ ) treated with IGSRT (Image-Guided Superficial Radiation Therapy). Dots represent censored events. 
Survival: Disease Free Survival (DFS) at 30.8 months was $100 \%$. At an average follow-up of 16.23 months, 92 of 93 patients were alive. One patient expired from unrelated causes while NED.

Table 3: Patient demographics at the time of treatment.

\begin{tabular}{|c|c|c|}
\hline \multicolumn{3}{|c|}{ Tumor Characteristics } \\
\hline \multicolumn{2}{|c|}{ Number of Lesions } & 133 \\
\hline \multirow{3}{*}{ Histopathology } & $\begin{array}{l}\text { BCC - Basal Cell } \\
\text { Carcinoma }\end{array}$ & 67 (50.4\%) \\
\hline & $\begin{array}{c}\text { SCC - Squamous Cell } \\
\text { Carcinoma }\end{array}$ & 17 (12.8\%) \\
\hline & $\begin{array}{l}\text { SCCIS - Squamous Cell } \\
\text { Carcinoma In Situ }\end{array}$ & $49(36.8 \%)$ \\
\hline \multirow{5}{*}{ Stage } & T0 & $49(36.8 \%)$ \\
\hline & $\mathrm{T} 1$ & $77(57.9 \%)$ \\
\hline & $\mathrm{T} 2$ & $7(5.3 \%)$ \\
\hline & T3 & $0(0 \%)$ \\
\hline & $\mathrm{T} 4$ & $0(0 \%)$ \\
\hline \multirow{4}{*}{ Lesion Size $(\mathrm{cm})$} & Mean & $1.04(\mathrm{SD}+/-0.63)$ \\
\hline & Median & 1 \\
\hline & Minimum & 0.11 \\
\hline & Maximum & $4.5^{*}$ \\
\hline
\end{tabular}

*SCCIS (Squamous Cell Carcinoma In Situ) all with full thickness atypia.

\section{Recurrence}

The only recurrence in this cohort was a stage I nodular BCC lesion located on the ear which recurred at 12.9 months. This lesion received 20 fractions at $50 \mathrm{kV}$ of 251.79 cGy for a total cumulative dose of 5035.80 cGy. The Time Dose Fractionation (TDF) number for this lesion was 85 . Treatment duration for this lesion was 1.64 months, which was within 1 standard deviation (SD) of the average treatment duration (1.56 months +/- 0.27 days). On follow-up 14 weeks after IGSRT completion, the lesion was noted as "well healed and no evidence of recurrence". Recurrence was detected 56 weeks later (12.9 months) and confirmed by biopsy with pathology showing nodular BCC. Salvage with excision and electrodessication was performed. Pathology after salvage, excision and ED\&C confirmed scar with no residual lesion identified.

\section{Cosmesis}

Cosmesis was felt to be almost entirely excellent and occasionally very good (VG) by the radiation oncologist and dermatologist with no fair or poor cosmesis.

\section{Toxicities}

Acute toxicities were graded using RTOG criteria (Table 4) [15] Table 5 shows the distribution by RTOG toxicity. All lesions had minimal or mild toxicity (RTOG $0,1,2$ ) with no lesions having severe or significant toxicity (RTOG 3,4$)$. There were no severe toxicities or complications requiring treatment interruption or delay.

Table 4: RTOG (Radiation Treatment Oncology Group) acute skin radiation toxicity [15].

\begin{tabular}{|c|c|}
\hline Grade & Signs/Symptoms \\
\hline 0 & No symptoms \\
\hline 1 & $\begin{array}{c}\text { Follicular, faint or dull erythema; epilation; dry } \\
\text { desquamation; decreased sweating }\end{array}$ \\
\hline 2 & $\begin{array}{c}\text { Tender or bright erythema, patchy moist desquamation; } \\
\text { moderate edema }\end{array}$ \\
\hline 3 & $\begin{array}{c}\text { Confluent, moist desquamation other than skin folds, pitting } \\
\text { edema }\end{array}$ \\
\hline 4 & Ulceration, hemorrhage, necrosis \\
\hline 5 & Death directly related to radiation effects \\
\hline
\end{tabular}

Table 5: Acute toxicities RTOG (Radiation Treatment Oncology Group) grades for lesions treated with IGSRT (Image-Guided Superficial Radiation Therapy).

\begin{tabular}{|c|c|}
\hline RTOG & Number of Lesions \\
\hline 0 & 1 \\
\hline 1 & 101 \\
\hline 2 & 31 \\
\hline 3 & 0 \\
\hline 4 & 0 \\
\hline
\end{tabular}

\section{Discussion}

There is presently very little modern data on the use of superficial radiotherapy for the treatment of NMSC. The American Academy of Dermatology (AAD) recommends superficial radiation therapy as a secondary treatment option reserved for special cases of NMSC $[16,17]$. This suggests that further research is necessary to establish the long-term efficacy and safety of SRT to establish that it provides comparable or acceptable cure rates in relation to MMS. The American Society for Radiation Oncology (ASTRO) analyzed the literature from May 1988 to June 2018 of studies that utilized radiotherapy (RT) for curative intent of BCC and CSCC lesions and concluded that there is limited modern randomized controlled trials (RCT) on RT and an absence of prospective RCT comparing NMSC treatment modalities [18]. Furthermore, there is even fewer data involving use of the newer technology of IGSRT, which this retrospective study reports.

In this retrospective study, IGSRT was found to be feasible, effective, safe, convenient, and achieves excellent/VG cosmetic outcomes. These results highlight the promise that IGSRT may be considered a first line alternative non-surgical option for the treatment of NMSC. These results are also consistent with the limited current published literature which support the use of 
SRT for the treatment of NMSC. Roth et al. [19] evaluated the effectiveness of SRT to treat 38 BCC and 113 SCC lesions located on the lower extremity in an elderly population with a mean age of 82.5. The follow-up for a majority of patients was $>2$ years, with $17 \%$ followed $<2$ years. The overall cure rate was found to be $97.4 \%$ with four lesions that recurred and successfully salvaged. Another recent study published by Roth et al., retrospectively evaluated the long-term safety and efficacy of using SRT to treat NMSC in 516 patients with 776 lesions (448 BCC and 328 SCC) [20]. Six recurrences were noted $(0.77 \%)$ with a KM local control rate of $98.9 \%$. at 7.1 years and the only minor long-term toxicity was hypopigmentation. The results from both of these studies are consistent with our findings using IGSRT.

Cognetta et al. [21] concluded that SRT was a viable non-surgical option for the treatment of NMSC with 5-year KM LC of $95.8 \%$ for BCC and $94.2 \%$ for SCC (invasive and in situ) in 1715 NMSC lesions located on the face and scalp from 1149 patients [21]. They also report a 2-year KM recurrence rate of $2 \%$ for BCC, $1.2 \%$ for invasive SCC, and $1.9 \%$ for SCCIS. The 5-year recurrence rate reported in Cognetta et al. [21] was compared to a 5-year recurrence rate of $1.0 \%$ for BCC and a 5-year recurrence rate of $2.6 \%$ for primary SCC treated with MMS. One difference between the regimen by Cognetta et al. [21] and ours is the fractionation regimen used. Most lesions in their series were treated with 5 fractions of 700 cGy for a total cumulative dose of 3500 cGy. In our study, we routinely used 20 fractions of approximately 255 cGy for a total cumulative dose in the range of $5100 \mathrm{cGy}$. Another difference in our study is the use of image guidance to determine the tumor depth before, during, and after treatment. Our results are similar in comparison to Cognetta et al. [21] with a slightly improved 2-year KM recurrence rate for SCC and SCCIS. We report a 2-year KM recurrence rate of $2.22 \%$ for BCC, $0 \%$ for invasive SCC and $0 \%$ for SCCIS. These early results concur with those of Cognetta et al. [21] with a suggestion that SRT may be potentially superior to MMS for SCC particularly at certain high risk regions, as one study reported the 2-year recurrence rate of SCC of the ear treated with MMS to be $5.7 \%$ (5 of 87 tumors) [22]. With longer follow-up this trend may continue to hold true, and is also suggested in another study (Yu et al. unpublished data). The reason for this potential superiority may be due to the routine use of a margin of "safety" (umbra) beyond the SCC lesion (typically $5-10 \mathrm{~mm}$ distal to the edge of the lesion), which is a wider margin than that for MMS as the latter is purposely designed to conserve as much tissue from surgical removal as possible.

Our study further demonstrates that superficial radiotherapy combined with an image-guided modality does not detract from its effectiveness and may in fact enhance its effectiveness. There appears to be additional benefits of using image guidance as it can allow visualization of tumor dimensions even under the surface of the skin for better targeting accuracy as well as the reliable assessment of tumor depth. This is akin to the advantages of using polarized dermoscopy to help elucidate a lesion that is only visualized on the surface. The measurement of tumor depth is important as it allows selection of the proper penetrating energy to adequately encompass the entire tumor. The proper selection of energy can improve cosmetic results as normal tissue that is left unperturbed maintains a better cosmesis. On the other hand, if the energy used is not sufficient to cover the entire tumor, this can potentially result in decreased local control. The only lesion that recurred in our study was treated with an energy of $50 \mathrm{kV}$ and had a relatively low TDF of 85. Our protocol currently increases the energy to $70 \mathrm{kV}$ or higher and also increases the TDF number for nodular BCC lesions and is anticipated to decrease failure rates going forward. Furthermore, ultrasound use can detect potential satellite lesions or unexpected deep involvement which may change the management from non-surgical to surgical recommendations. The principles of image guidance in improving radiotherapy outcome has been documented in various previous studies on different sites of disease in the radiation treatment of neoplasms [23-25].

This study suggest IGSRT has advantages over surgery for NMSCs, especially lesions located in cosmetically sensitive areas, such as the head and neck where a majority of NMSC lesions occur, where cosmetic and functional outcome is paramount. Having a NMSC treated in a vulnerable area, such as the face, is often associated with patient anxiety and concern about the cosmetic outcome especially with surgery [10]. In this study IGSRT appears to be able to achieve this without compromise in the control rate. Current research indicates after an individual's first NMSC, the risk for another one is $40.7 \%$ within 5 years [26]. For individuals diagnosed with a non-first NMSC the 5-year probability of another NMSC increases to $82.0 \%$. Many patients suffer surgical fatigue from having multiple surgical procedures on an ongoing basis. In patients who may fear or decline surgery, IGSRT provides a viable and effective non-surgical option for these patients. Additionally, IGSRT is advantageous to treat NMSC in areas prone to poor wound healing, bleeding and infections (ie. below the knee, or very mobile or exposed portions of the skin). For patients who are on anticoagulation, the treatment can be given without suspension of the use of anticoagulants, minimizing the risk of stroke or thrombolytic events. Patients are very pleased with the results and availability of this non-surgical option and frequently request this option for additional lesions that occur after their first experience. From the providers' experience with IGSRT, there is improved patient compliance with treating their skin cancers versus ignoring them given this additional option.

Limitations of this study include that it is a retrospective observational study on a small number of lesions with less than 5-year follow-up. No randomization was employed. A matched cohort analysis may be beneficial to compare the results of 
this modality to other surgical techniques as randomization to a surgical versus non-surgical treatment may be ethically or practically difficult to accomplish. Another drawback is the use of provider reporting of recurrences, which introduces a potential underreporting bias. Further follow-up with a minimum of 2-year and preferably 5-year follow-up is warranted.

\section{Conclusion}

Office based IGSRT is feasible, safe, and easily tolerable. On early follow-up, this 20 -fraction regimen is highly effective with an overall absolute LC of $99.2 \%$, and an overall KM LC of $98.95 \%$ at 30.8 months, with a $100 \%$ DFS. It achieves excellent or very good cosmesis and patients are very happy with the results and availability of this nonsurgical option. IGSRT is an attractive non-invasive therapeutic option for NMSC and provides another valuable tool for dermatologists. Longer follow-up is underway.

\section{Acknowledgments for Grants and Technical Support}

The authors would like to acknowledge the following people who were instrumental in collecting and extracting data: Nick Natalizio, Krupal Patel, and Songzhu Zhang. We extend a special thanks to Songzhu Zhang for his invaluable assistance in statistical analyses and curve generation.

\section{Conflicts of Interest}

We would like to disclose the following potential conflicts of interest: Dr. Lio Yu is the National Radiation Oncologist for SkinCure Oncology and has received research, speaking and/or consulting support from SkinCure Oncology. He has served on an advisory board for Bayer Pharmaceuticals previously. Raymond Beers, M.S works for Astarita Associates, which SkinCure Oncology contracts with to provide physics support to Laserderm Dermatology of Smithtown. Dr. Donna Serure has no conflicts of interest to disclose. Mairead Moloney has no conflicts of interest to disclose.

\section{Funding Sources}

None.

\section{Compliance with Ethics Guidelines}

The authors adhered to the principles established in the Federal Policy for the Protection of Human Subjects, referred to as the "Common Rule," as well as the pertinent sections of the Helsinki Declaration and its amendments. The data have been de-identified for use in this study.

\section{References}

1. Apalla Z, Lallas A, Sotiriou E, Lazaridou E, Ioannides D (2017) Epidemiological trends in skin cancer What Does the Future Hold. Dermatol Pract Concept 7(2): 1-6.
2. Rong Y, Zuo L, Shang L, Bazan JG (2015) Radiotherapy treatment for nonmelanoma skin cancer. Expert Rev Anticancer Ther 15(7): 765-776.

3. https://www.cancer.org/content/dam/cancer-org/research/cancerfacts-and-statistics/annual-cancer-facts-and-figures/2020/cancerfacts-and-figures-2020.pdf.

4. Fahradyan A, Howell A, Wolfswinkel E, Tsuha M, Sheth P, et al. (2017) Updates on the Management of Non-Melanoma Skin Cancer (NMSC). Healthcare 5(4): 82.

5. Garcovich S, Colloca G, Sollena P, Andrea B, Balducci L, et al. (2017) Skin cancer epidemics in the elderly as an emerging issue in geriatric oncology. Aging Dis 8(5): 643-661.

6. Rogers HW, Weinstock MA, Feldman SR, Coldiron BM (2012) Incidence estimate of nonmelanoma skin cancer (keratinocyte carcinomas) in the us population, 2012. JAMA Dermatology151(10): 1081-1086.

7. Alam M, Armstrong A, Baum C (2018) Guidelines of care for the management of cutaneous squamous cell carcinoma. J Am Acad Dermatol 78(3): 560-578.

8. Nestor MS, Berman B, Goldberg D, Cognetta AB, Gold M, et al. (2019) Consensus Guidelines on the Use of Superficial Radiation Therapy for Treating Nonmelanoma Skin Cancers and Keloids. J Clin Aesthet Dermatol 12(2): 12-18.

9. Linos E, Parvataneni R, Stuart SE, Boscardin WI, Landefeld CS, et al. (2013) Treatment of nonfatal conditions at the end of life: Nonmelanoma skin cancer. JAMA Intern Med 173(11): 1006-1012.

10. Zagrodnik B, Kempf W, Seifert B, Müller B, Burg G, et al. (2003) Superficial Radiotherapy for Patients with Basal Cell Carcinoma: Recurrence Rates, Histologic Subtypes, and Expression of p53 and Bcl-2. Cancer 98(12): 2708-2714.

11. Patel R, Strimling R, Doggett S, Willoughby M, Miller K, et al. (2017) Comparison of electronic brachytherapy and Mohs micrographic surgery for the treatment of early-stage non-melanoma skin cancer: a matched pair cohort study. J Contemp Brachytherapy 9(4): 338-344.

12. McGregor S, Minni J, Herold D (2015) Superficial radiation therapy for the treatment of nonmelanoma skin cancers. J Clin Aesthet Dermatol 8(12): $12-14$

13. Amin MB, Edge S, Greene F, Byrd DR, Brookland RK, et al. (2017) AJCC Cancer Staging Manual. $8^{\text {th }}$ edn. Springer, New York, USA.

14. https://www.nccn.org/professionals/physician_gls/pdf/squamous.pdf.

15. Cox JD, Stetz JA, Pajak TF (1995) Toxicity criteria of the Radiation Therapy Oncology Group (RTOG) and the European organization for research and treatment of cancer (EORTC). Int J Radiat Oncol Biol Phys 31(5): 1341-1346.

16. https://www.aad.org/\%0AForms/Policies/Uploads/PS/PS-Superficial \%2520Radiation\%25\%0A20Therapy.pdf.

17. Christopher KB, Alam M (2018) Guidelines of care for the management of basal cell carcinoma. J Am Acad Dermatol 79(5): e101.

18. Likhacheva A, Awan M, Barker CA, Bhatnagar A, Bradfield L, et al. Definitive and Postoperative Radiation Therapy for Basal and Squamous Cell Cancers of the Skin: Executive Summary of an American Society for Radiation Oncology Clinical Practice Guideline. Pract Radiat Oncol 10(1): 8-20.

19. Roth WI, Shelling M, Fishman K (2019) Superficial Radiation Therapy: A Viable Nonsurgical Option for Treating Basal and Squamous Cell Carcinoma of the Lower Extremities. J Drugs Dermatol 18(2): 130-134.

20. Roth W, Beer RE, Iyengar V, Bender T, Raymond I (2020) Long-Term Efficacy and Safety of Superficial Radiation Therapy in Subjects With 
Nonmelanoma Skin Cancer: A Retrospective Registry Study. J Drugs Dermatol 19(2): 163-168.

21. Cognetta AB, Howard BM, Heaton HP, Stoddard ER, Hong HG, et al. (2012) Superficial x-ray in the treatment of basal and squamous cell carcinomas: A viable option in select patients. J Am Acad Dermatol 67(6): 1235-1241.

22. Silapunt S, Peterson SR, Goldberg LH (2005) Squamous cell carcinoma of the auricle and Mohs micrographic surgery. Dermatol Surg 31(11 Pt 1): $1423-1427$

23. Western C, Hristov D, Schlosser J (2015) Ultrasound Imaging in Radiation Therapy: From Interfractional to Intrafractional Guidance. Cureus 7(6): e280.
24. Sayan M, Vergalasova I, Hard D, Wrigth H, Archambault J, et al. (2019) Daily localization of partial breast irradiation patients with threedimensional ultrasound imaging. Radiat Oncol J 37(4): 259-264.

25. Bohrer M, Schröder P, Welzel G (2008) Reduced rectal toxicity with ultrasound-based image guided radiotherapy using BAT (B-mode acquisition and targeting system) for prostate cancer. Strahlentherapie Onkol 184(12): 674-678.

26. Wehner MR, Linos E, Parvataneni R, Stuart SE, Boscardin WJ, et al. (2015) Timing of subsequent new tumors in patients who present with basal cell carcinoma or cutaneous squamous cell carcinoma. JAMA Dermatology 151(4): 382-388. 